\title{
Energy balance analysis in non linear dynamic equivalent systems.
}

\author{
Iturregui Arranz Carlos ${ }^{1, *}$, Soria Herrera Jose Manuel $^{1}$, Muñoz Díaz Ivan ${ }^{1}$, García Palacios Jaime Higinio ${ }^{1}$. \\ ${ }^{1}$ Department of Continuum Mechanics and Theory of Structures, Escuela Técnica Superior de Ingenieros de Caminos, \\ Canales y Puertos- Technical University of Madrid, 28040 Profesor Aranguren, Spain
}

\begin{abstract}
The aim of the paper is to present a critical analysis for nonlinear dynamic vibrations. It is applicable to single degree of freedom -SDOF- of reinforced concrete -RC- structures, revealing its multi degree of freedom -MDOF- performance, showing contrast using the balance of energy, using six accelerograms based on type I and II spectrum, according with Eurocode- 8 . The degradation curve was obtained applying a new formulation, based on the system work and complementary work, into the pushover analysis. A new method incorporating the Bouc-Wen-Baben-Noori theory and global damage was used for the analysis, adding relevance to the: energy balance in its dissipative part, analysis of the structure's fundamental parameters, relation's effective period, equivalent damping and global ductility.

The powers, energies and works developed are analyzed, creating a precise balance since energy enters selectively. Hence, an equivalent damping containing a viscous and hysteretic part is predictable, accordingly to the variation of the building's nonlinear properties. Evaluation of the adequateness and safety level are also obtainable. The controlled parameters contrasted with the balance predicts the structure's MDOF situation, at any moment related with seismic events. This methodology can be used to stablish a systematic control of nonlinearities for other structural schemes.
\end{abstract}

\section{Introduction.}

In an SDOF system, the general model, normalized to mass, is:

$$
\ddot{x}(t)+g(\dot{x}, x)=f(t)
$$

In this equation the first term corresponds to the inertial forces due to the acceleration of the system, the second $g(x, x)$ to the forces of restoration and loss of energyvelocity by damping, and the right hand side is the external action $f(t)$

In case of a seismic action the linear equation is:

$$
m x(t)+c x(t)+k x(t)=-m a(t)
$$

In the case of a non-linear system, the energy balance can be established in a similar way:

$$
m \ddot{x}(t)+\int_{0}^{t} G(t-\tau) \dot{x}(\tau) d \tau+f(x, t)=-m a(t)
$$

With this analysis, it is possible to do a general balance of the system, which will be extended to the MDOF systems, since as demonstrated by Akiyama the energy input of an MDOF system is the sum of the energy input of the SDOF that composes it [1].

$$
W_{e k}+W_{d}+W_{e}+W_{p}=E
$$

Where $W_{e k}, W_{d}, W_{e}, W_{p}$ are the kinetic, viscous damping, elastic and plastic work develop by the system's forces, and $E$ is the energy input.
As an example, 6 synthetic accelerograms, created with the software acelsin [2], will be used. In the figures 1.1. and 1.2 are shown the numbers 1 and 4 .

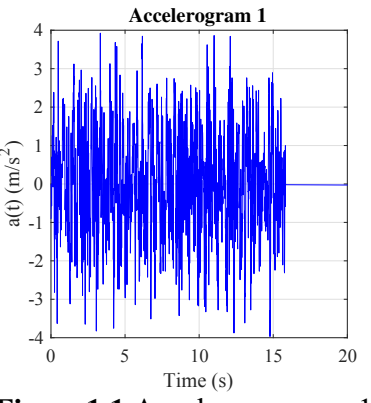

Fig 1.1.Accelerogram 1 Example of application. Spectrum type II.

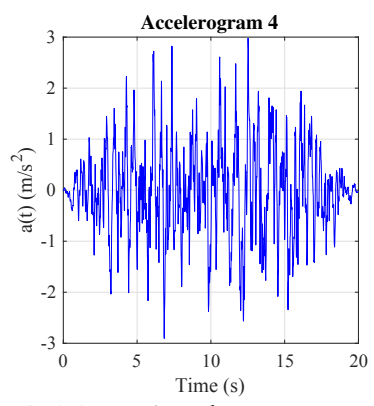

Fig1.2. Accelerogram 4 Example of application. Spectrum type I.
In the figure 2 it is shown the structure under analysis:

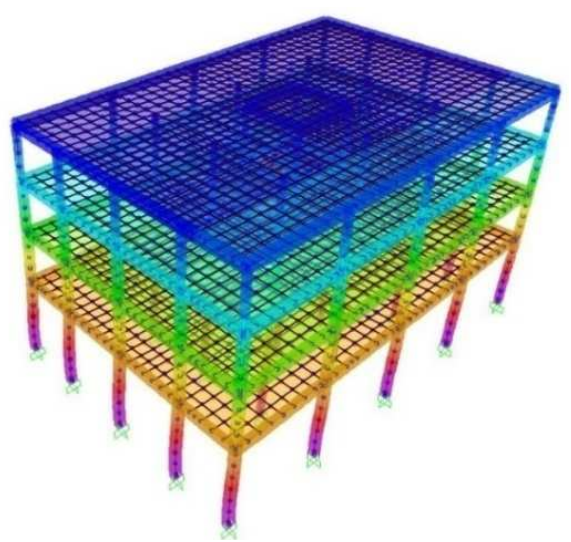

Fig 2. Building GF+3F. $\mathrm{m}^{*}=2518$ t. $\Gamma=1.2956$. According with EC-8 [3]. 


$$
\mathrm{K}_{\mathrm{O}}=74074 \frac{k N}{m} \mathrm{~T}_{\text {eqSDOF }} *=1.135 \mathrm{sec} .
$$

The building has been designed conventionally with the ETABS program. [4] and EC 2 and 8. The symbols $\mathrm{m}^{*}$, $\Gamma, \mathrm{K}_{\mathrm{O}}$ and $\mathrm{T}_{\text {eqSDOF }}{ }^{*}$ are refer to those codes.

\section{Relevant parameters to consider in the energy evaluation of non linear systems in concrete structures.}

\subsection{Damage.}

The mechanical properties of damaged differs from those initially conceived in the elastic design. This paper will concentrate the inelastic parameters, besides the lateral deformation in the case of concrete buildings considering they are indicative of the structure's damage.

\subsection{Effective period- $T_{e}$.}

- Akiyama proposed a general expression for an SDOF system as follows:

$T_{e}=\psi(\mu) T_{o}\left(\frac{\mu}{q}\right)^{0.5}$

$\mu$ is the coefficient of ductility, $\mathrm{q}$ is the instantaneous force normalized to the yield shear force. $\psi(\mu)$ is a function of ductility and the period of the undamaged structure.

- Kowlasky, Calvi and Priestley [5] proposed a formulation for a bilinear inelastic solid, whose value is

$T_{e}=T_{o}\left(\frac{\mu}{1+r(\mu-1)}\right)^{0.5}$

$r$ is the ratio of inelastic to elastic stiffness in the hardening section, and $\mu$ is the coefficient of deformational ductility of the system, that is the ratio between maximum displacement and elastic yield displacement.

In this paper is proposed a formula based on the work and complementary work developed by the system in the general case of inelasticity, not necessarily linear, developed by any SDOF or equivalent SDOF system.

The figure 3 shows building push over curve, being the shear force $\mathrm{F}$ and the displacement $\Delta$.

Then, with this notation:

$W=\int_{0}^{\Delta} F(\Delta) d \Delta$ and $W^{*}=\int_{0}^{F} \Delta(F) d F$ with $\mu(\Delta)=\frac{\Delta}{\Delta_{y}}, T_{e}=T_{o}\left(1+\frac{W-W^{*}}{W+W^{*}} \mu\right)^{0.5}$ (7)

The factor that regulates the function is $f=\frac{W-W^{*}}{W+W^{*}}$, which is the ratio between the difference and the sum of work and complementary work of a structural system.

The figure 4 shows the frequency evolution with the displacement, obtained with the secant period proposed in the formula (7).

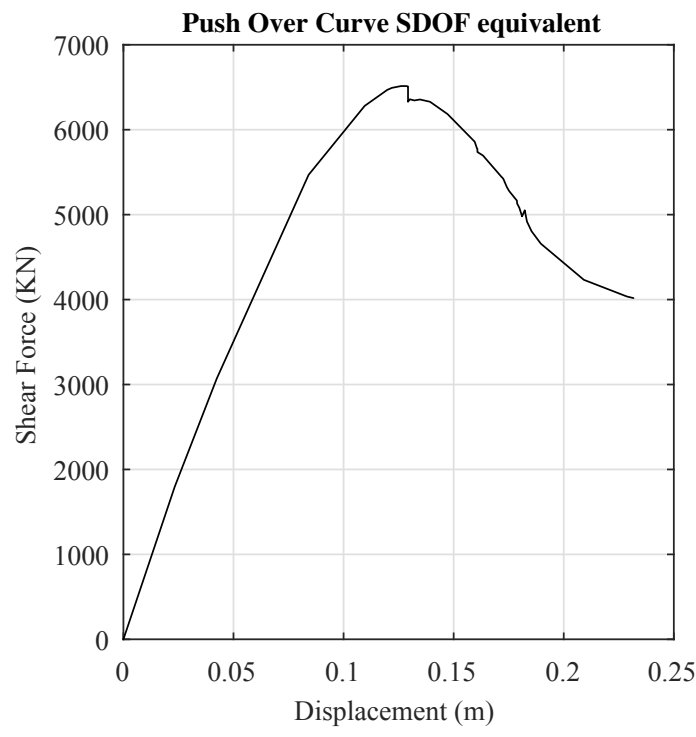

Fig 3. Push-Over curve for a $\mathrm{GF}+3 \mathrm{~F}$ building

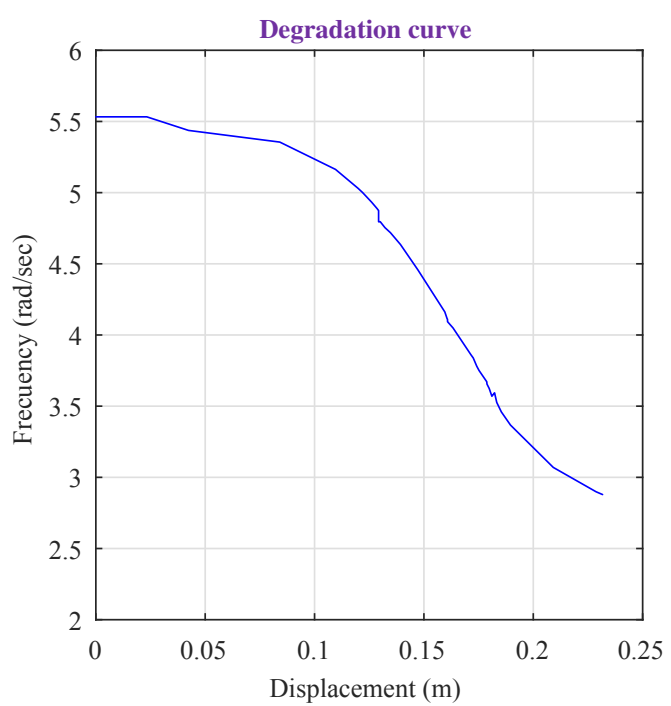

Fig 4. Degradation curve obtained with equation 7. Application to a $\mathrm{GF}+3 \mathrm{~F}$ building with first natural frequency $5.543 \mathrm{rad} / \mathrm{s}$ for the SDOF equivalent.

\subsection{Inelastic damping.}

The energy dissipation is one of the most relevant aspects of the behaviour of a structure versus external action in 
civil construction, especially when the load is a base acceleration.

Akiyama [6] made numerous studies in elastic-perfectly plastic systems, arriving at a conclusion on the hysteretic work developed by the system.

Hysteretic damping coefficient is defined by Jacobsen's [7] as:

$$
\zeta_{\text {eq }}=\frac{1}{4 \pi} \frac{A_{\text {hyst }}}{F_{0} A_{0}}
$$

It is a ratio between hysteretic energy in one complete loop in comparation with the maximum elastic energy.

Gulkan and Sozen [8] proposed an evolution of

$$
\text { Jacobsen's methodology } \zeta_{e q}=\frac{T_{e q} \int_{0}^{t} a(t) \dot{x}(t) d t}{4 \pi \int_{0}^{t} \dot{x}^{2}(t) d t}
$$

The formulation is critically analysed:

Integrating in (2) the powers:

$\int_{0}^{t} a(t) \dot{x}(t) d t=\frac{E}{m}$ and

$\int_{0}^{t} \dot{x}^{2}(t) d t=\frac{W_{d}}{c}=\frac{m W_{d}}{2 \xi_{0} \omega}$, being $\xi_{0}$ the elastic viscous

damping coefficient.

Substituting in the equation of Gulkan and Sozen:

$$
\zeta_{e q}=\frac{T_{e q} \frac{E}{m}}{4 \pi \frac{W_{h}}{c}}=\frac{T_{e q} E c}{4 \pi m W_{h}}=\frac{E \zeta_{o} \omega}{2 \pi W_{h}} T_{e q}=\frac{E}{W_{h}} \zeta_{o}(10)
$$

In elastic system $W_{d} \approx E$, so $\zeta_{e q} \approx \zeta_{o}$

In the case of an inelastic system, the same formulation can also be considered, simply replacing c by $\mathrm{c}(\omega)$, although some precision may be lost.

Other formulations have been developed by Kowalsky, Calvi and Priestley [5] depending on the ductility required by the system, with or without period dependence.

$\zeta_{e q}=\zeta_{0}+a\left(1-\frac{1}{\mu^{b}}\right)\left(1+\frac{1}{\left(T_{e q}+c\right)^{d}}\right) \frac{1}{N}$

This formulation greatly improved the one raised by Gulkan and Sozen, since it takes into account the degree of damage of the structure as well as damage model, with the parameters $\mathrm{a}, \mathrm{b}, \mathrm{c}$ and $\mathrm{d}$.

All aspects of this point are schematically summarized in the figure 5 , by superposition of the last 4 seconds in the
Inelastic Time History Analysis (ITHA) with linear and Bouc-Wen-Baber-Noori (BWBN) theory (look 3.2):

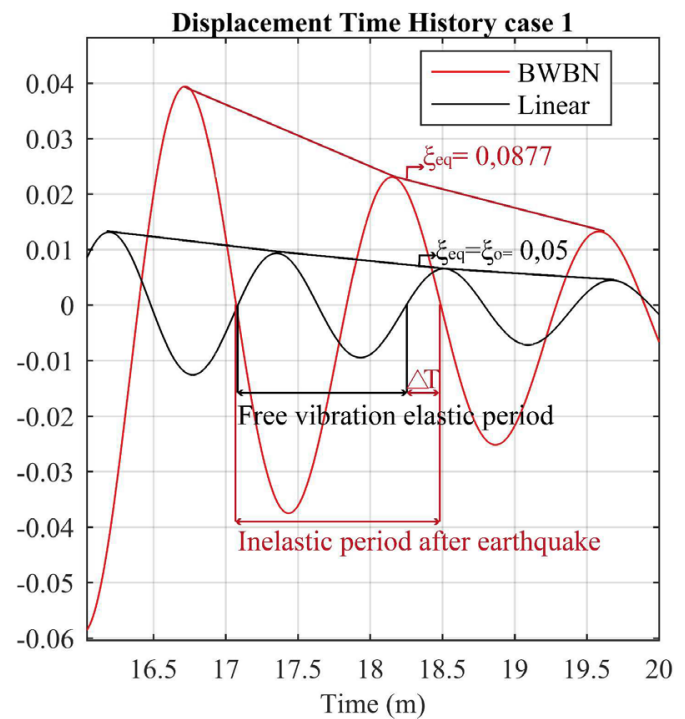

Fig 5. Schemes of inelastic quasi-period, equivalent damping caused by damage to concrete structures. Example figure 1.1. Case I.

The energy balance based design is based on, once the inelastic energy input is defined, the formulation of the viscous and plastic working developed by the structural system.

In the case of an unknown external action clearly known as a project element, the energy balance based design becomes a much more stable than forces method.

In addition to this, the energy input can be established in the balance if the equivalent damping is proportional to the viscous work.

\section{Analysis of nonlinear equivalent models.}

\subsection{Nonlinear model with accumulated degradation associated to an incremental push curve. Analysis of damping problem and effective period of vibration.}

Over the last few years the incremental push-over procedure has been implemented in different regulations, especially in seismic design standards. It consists of obtaining a SDOF substitute structure from the incremental monotonous push until breaking, it is then analyzed in a static way.

Many studies of this methodology have been done by Chopra [9], although they have been restricted to the adaptive push over methodology.

In concrete structures it could be assumed that there is a monotonous decreasing of the natural frequency of 
vibration, since, except the crack close by loop change, a cracked piece can not gain vibration frequency. Even in the case of close of cracks the only "distortion" is the lack of symmetry of the load loop.

From the non-linear static analysis curve it is possible to obtain the Inelastic Time History Analysis (ITHA), in comparation with the elastic Time History Analysis (THA) with the equation:

$$
\ddot{x}(t)+2 \xi_{o} \omega(x, t) \dot{x}(t)+\omega^{2}(x, t) x(t)=-a(t)
$$

In case of THA $\omega(x, t)=\omega_{0}$, which is supposed constant value.

For to obtain the ITHA an algorithm has been created in Matlab using the push-over curve (fig 3), so that the frequency is derived from the degradation curve (fig 4) in function of the secant inelastic period, obtained with (7). The results shown for case 1 and 4 are representative of all 6 .

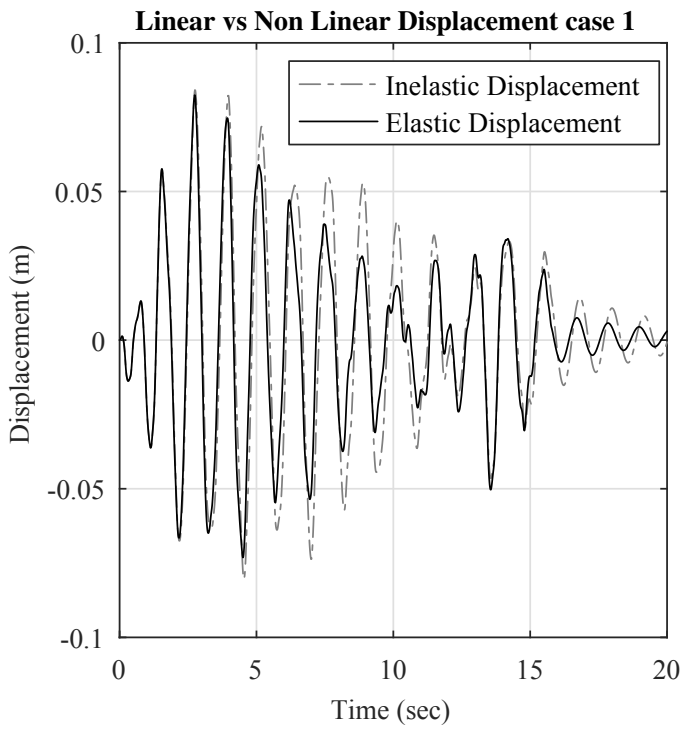

Fig 6. THA and ITHA of displacements case 1.

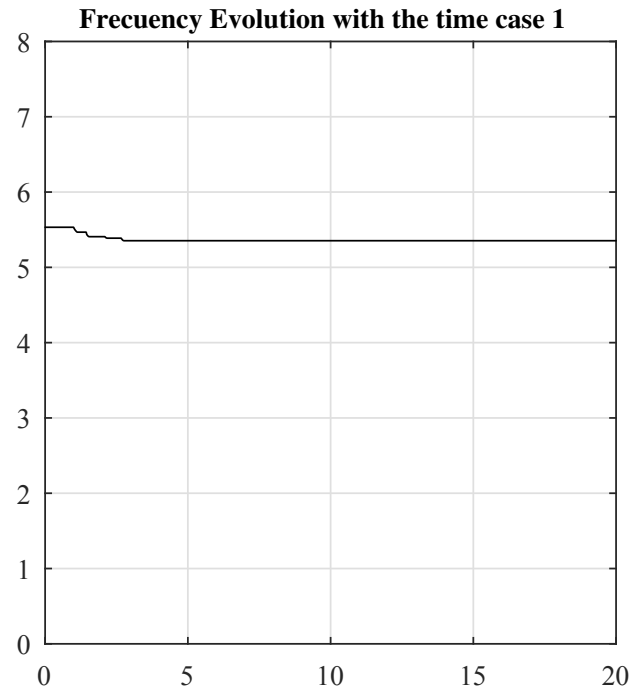

Fig 7. Evolution of circular frequency during the earthquake, case 1. (Y-Frecuency rad/s); (X,time)
In the case of type II spectrum accelerograms the degradation is quite low (fig 7). In spite of this, the parameters are observed quite well. Conversely, in type I spectrum, the degradation is much more pronounced (fig 9).

It is expected to have more damage and inelasticity in accelerograms obtained with type I spectrum, even if the acceleration is smaller, precisely because the energy introduced in the system is greater for periods over $0.7 \mathrm{~s}$, like it is shown in the present paper example.

Table 1. Maximum values of the spectrums type I and II. EC-8. $0.25 \mathrm{~g}$, soil IV. $\mathrm{S}_{\mathrm{v}}$ is cuasi-spectrum velocity.

\begin{tabular}{|c|c|c|c|}
\hline Spectrum & $\mathrm{S}_{\mathrm{a}}\left(\mathrm{m} / \mathrm{s}^{2}\right)$ & $\mathrm{S}_{\mathrm{v}}(\mathrm{m} / \mathrm{s})$ & $\mathrm{S}_{\mathrm{d}}(\mathrm{m})$ \\
\hline $\mathrm{I}$ & 8.268 & 1.05 & 0.335 \\
\hline II & 11.02 & 0.562 & 0.1 \\
\hline
\end{tabular}

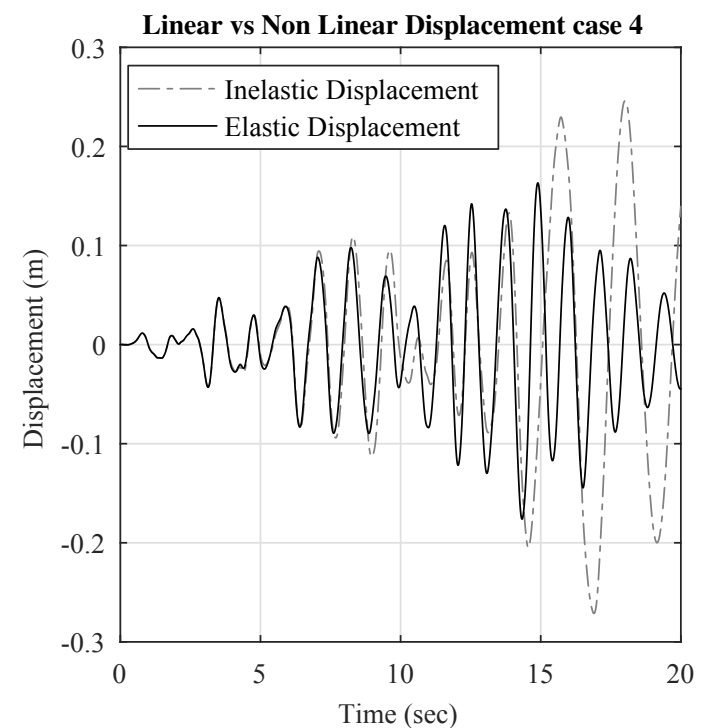

Fig 8. THA and ITHA of displacements case 4.

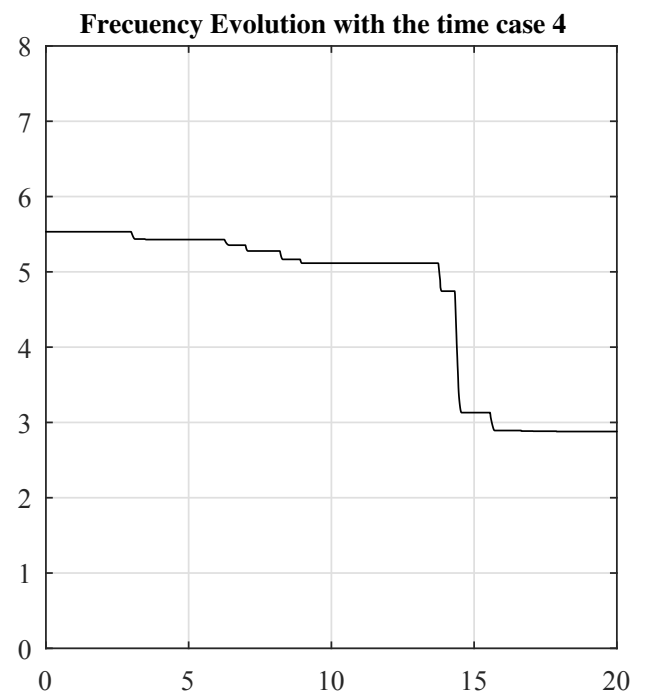

Fig 9. Evolution of circular frequency during the earthquake, case 4. (Y-Frecuency $\mathrm{rad} / \mathrm{s})$; (X,time) 


\subsection{Bouc Wen Baber Noori (BWBN).}

In the BWBN the general equation of the SDOF model is established as follow:

$$
\begin{aligned}
& \ddot{x}(t)+2 \xi_{o} \omega_{o} \dot{x}(t)+\alpha \omega_{o}^{2} x(t)+\ldots . \\
& \ldots . .+(1-\alpha) \omega_{o}^{2} z(t)=-a(t)
\end{aligned}
$$

The parameters has been adapted according with [10] and [11]; the last term of the power integrated equation is the Hysteretic Energy.

Tabel 2. Summary of maximum abs displacements (m).

\begin{tabular}{ccclcl} 
TH & Spectrum & Elastic & Push & BWBN & DDBD \\
\hline 1 & II & 0.08420 & 0.08420 & 0.06260 & 0.04905 \\
2 & I & 0.14560 & 0.19080 & 0.15360 & 0.16453 \\
3 & II & 0.08510 & 0.07910 & 0.06710 & 0.04151 \\
4 & I & 0.17610 & 0.27130 & 0.01497 & 0.13063 \\
5 & II & 0.09170 & 0.09290 & 0.07890 & 0.04126 \\
6 & I & 0.16100 & 0.37110 & 0.20880 & 0.13507 \\
\hline
\end{tabular}

Note: The displacements according with DDBD methodology are explained in 3.3 .

\subsection{Methods of synthesis. Direct Displacement Based Design (DDBD) example.}

Generally the interest to know the evolution during the time of the parameters that regulate the behaviour of a structural system is irrelevant This lack of interest promotes certain methods such as the dynamic modal spectral analysis and the direct displacement design method (DDBD), which overall, have had a great implementation in professional practice.

The DDBD method is summarised by calculating an equivalent SDOF structure with an elastic plus an inelastic phase. In a reinforced concrete frame building:

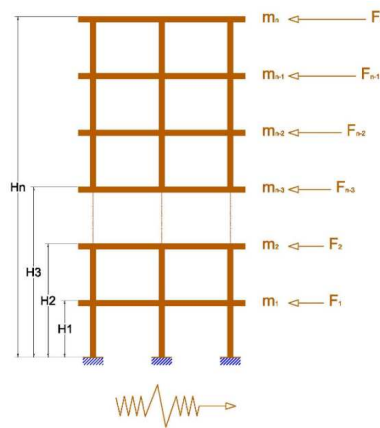

Fig 10.1 MDOF real Structure

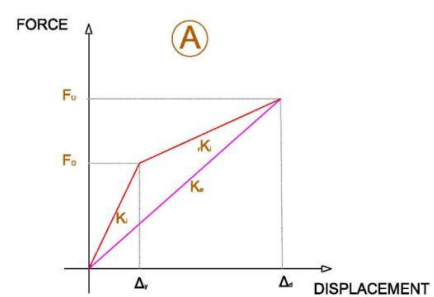

Fig 10.3 DDBD Force-Balance Scheme.

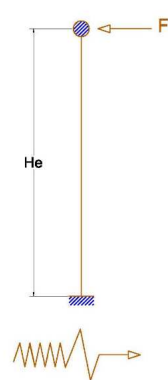

Fig 10.2 Equivalent SDOF scheme

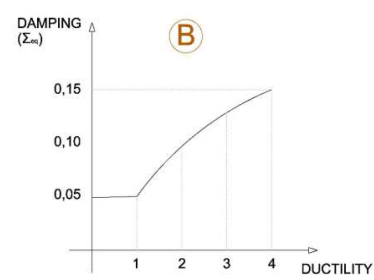

Fig 10.4. R DampingDuctility curve.
$\Delta_{i}=\delta_{i}\left(\frac{\Delta_{c}}{\delta_{c}}\right)=\left(\frac{\Delta_{c}}{\delta_{c}}\right)\left[\frac{4}{3}\left(\frac{H_{i}}{H_{n}}\right)\left(1-\frac{H_{i}}{4 H_{n}}\right)\right]$

The equivalent displacement is: $\Delta_{d}=\frac{\sum_{1}^{n} m_{i} \Delta^{2}{ }_{i}}{\sum_{1}^{n} m_{i} \Delta_{i}}$

The equivalent mass is: $m_{e}=\frac{\sum_{1}^{n} m_{i} \Delta_{i}}{\Delta_{d}}$

The equivalent height: $H_{e}=\frac{\sum_{1}^{n} m_{i} \Delta_{i} H_{i}}{\sum_{1}^{n} m_{i} \Delta_{i}}$

Whenever the equivalent period $T_{e}$ is known the parameters of the system could be fixed:

Equivalent Rigidity: $K_{e}=\frac{F}{\Delta_{d}}=\frac{4 \pi^{2} m_{e}}{T^{2}{ }_{e}}$

The equivalent period of the system is obtained by introducing the deformation demand of the equivalent system $\Delta_{d}(15)$ in the spectrum, based on equivalent damping. The method introduces some conceptual improvements such as the association of a displacement ductility proportionally to the damage (fig 10.4) and coherence for the damping balance (11).

In this case, the building $\mathrm{GF}+3 \mathrm{~F}$ is introduced in its equivalent SDOF response. Using the formulation of the energy balance the elastic work part is:

$W_{e}=K_{o} \frac{\Delta^{2} d y}{2}$

And the plastic work:

$W_{p}=\frac{K_{o} \Delta_{d y}+K_{e} \Delta_{d}}{2}\left(\Delta_{d}-\Delta_{d y}\right)$

In case of considering an inelastic section without hardening $W_{p}=F\left(\Delta_{d}-\Delta_{d y}\right)=F \Delta d$

whereby it is possible to obtain the increment of plastic displacement associated with the energy contributing to damage, deducing the elastic component.

Tabel 4. Summary of energy balance DDBD.

\begin{tabular}{llcllll} 
TH & Spec & $\mathbf{E}_{\mathbf{W}} \mathbf{W}_{\mathbf{d}}$ & $\mathbf{W}_{\mathbf{e}}$ & $\mathbf{W}_{\mathbf{p}}$ & $\boldsymbol{\Delta}_{\mathbf{d}}$ & Total \\
\hline 1 & II & 0.130 & 0.597 & 0.000 & 0.000 & 0.049 \\
2 & I & 0.881 & 0.597 & 0.284 & 0.060 & 0.165 \\
3 & II & 0.093 & 0.597 & 0.000 & 0.000 & 0.042 \\
4 & I & 0.719 & 0.597 & 0.122 & 0.026 & 0.131 \\
5 & II & 0.092 & 0.597 & 0.000 & 0.000 & 0.041 \\
6 & I & 0.741 & 0.597 & 0.144 & 0.030 & 0.135 \\
\hline
\end{tabular}




\section{Conclusions.}

The substitution of a MDOF system for a SDOF one is very common to simplify the seismic design projects. In this sense, due to the natural dispersion derived in the formulation and the action, the energy balance represents a parameter of fundamental contrast for the methodology of nonlinear vibrations, whatever its formulation may be. In particular, in the case of an equivalent SDOF system in concrete structures, the energy balance allows it to be to critically discussed and contrasted with the suitability between actions, inelastic response, damage, damping and effective vibration period.

This paper has shown the following aspects:

- A formulation has been developed to obtain the secant period of a structural system from the curve derived from the nonlinear static analysis, general form and including geometric and material nonlinearity. This formulation, based on the energy balance of the shear-displacement curve, may be more adequate than that which binds the modal mass, as it evolves during the development of inelasticity. This does not happen in the DDBD method, which always implies the same inelastic profile, with only the magnitude varying.

- It has been shown how, with some care in the parameter delimitation which regulate the concrete damage model, it is possible to evaluate with a high degree of accuracy its values, even in extreme cases where is not considered the energy dissipated in hysteresis cycles.

The energy balance, despite the difficulties of its approach, with a correct definition and calibration, can suppose a fundamental tool for professional practice in nonlinear vibrations and specifically in seismic engineering, as they are currently introduced in some design regulations.

\section{ACKNOWLEDGEMENTS}

The authors acknowledge the financial support provided by Ministry of Economy and Competitiveness (Government of Spanish Kingdom) by funding the Research Project REVES-P (DPI2013-47441). The authors also acknowledge the partial financial support provided by the Research Net VIBRASTRUNET (BIA2015-71942-REDT).

\section{References}

[1] H. Akiyama, "Collapse modes of structures under strong motions of earthquake," Ann. Geophys., vol. 45, no. 6, pp. 791-798, 2002.

[2] Don Jose Antonio Agudelo Zapata, User manual of Acelsin. Synthetic accelerograms. Granada (Spain)., 2017.

[3] Comité Técnico AEN/CTN 140 Eurocódigos estructurales, "Eurocode 8: Design of structures for earthquake resistance. Part 1: General $r$ ules, seismic actions and rules for buildings," p. 208, 2006.

[4] Computers and Structures Inc., "CSi Analysis Reference Manual,” p. 556, 2016.
[5] T. J. Sullivan, G. M. Calvi, and M. J. N. Priestley, "Initial Stiffness Versus Secant Stiffness in Displacement Based Design," 13th World Conf. Earthq. Eng., no. 2888, 2004.

[6] H. Akiyama, Metodología de proyecto sismorresistente de edificios basada en el balance energético. Reverté, 2003.

[7] S. Superiori and S. Pavia, "Reduction of Seismic Risk Equiva L Ent Viscous Damping Equations for Direct," 2004.

[8] R. Zaharia and F. Taucer, "Equivalent period and damping for EC8 spectral response of SDOF ring-spring hysteretic models," Eur. Comm. Jt. Res. Centre, Sci. Tech. Reports, vol. JRC 45403, no. m, 2008.

[9] A. K. Chopra and R. K. Goel, “A modal pushover analysis procedure for estimating seismic demands for buildings," vol. 582, no. January 2001, pp. 561-582, 2002.

[10] P. Sengupta and B. Li, "Modified Bouc-Wen model for hysteresis behavior of RC beamcolumn joints with limited transverse reinforcement," Eng. Struct., vol. 46, pp. 392406, 2013.

[11] A. E. Charalampakis and V. K. Ã. Koumousis, "ARTICLE IN PRESS On the response and dissipated energy of Bouc - Wen hysteretic model," J. Sound Vib., vol. 309, pp. 887-895, 2008. 